Ergebnisse der limnologischen Untersuchungen der Oberengadiner Seen

Von Dr. Marianne Bosli-Pavoni, EAWAG, Dübendorf 


\title{
Ergebnisse der limnologischen Untersuchungen der Oberengadiner Seen
}

\author{
Von Dr. Marianne Bosli-Pavoni, EAWAG, Dübendorf
}

\section{Einleitung}

Im Zusammenhang mit der Internationalen Arbeitsgemeinschaft «Donauforschung» und der Untersuchung des Inns als Nebenfluss der Donau erhielt die Limnologische Abteilung der EAWAG den Auftrag, Proben aus den Oberengadiner Seen zu holen und den Teilnehmern der 13. Tagung eine knappe Darstellung der limnologischen Merkmale dieser Seen zu vermitteln.

Die Seen des Oberengadins, bevorzugt durch ihre einzigartige geographische Lage, sind aus der Belletristik und als touristischer Anziehungspunkt weltbekannt geworden. Von Limnologen jedoch sind sie bis heute nur sporadisch untersucht worden. Dies ist wahrscheinlich auf die Vorstellung zurückzuführen, dass Bergseen a priori sauber sind. Andererseits mag der beträchtliche Arbeitsaufwand einer einigermassen gründlichen Probenahme und Analyse hemmend gewirkt haben. Vereinzelt liegen chemische Daten vor [15, 16], ferner erforschte Borner [2] die Bodenfauna des St.Moritzer Sees, und Bachmann [1] und MEssikommer [5] analysierten das Phytoplankton mit Hilfe von Netzzügen.

Ziel unserer Untersuchungen war, anhand einiger Probeserien den biologischen und chemischen Zustand der Seen zu erfassen. Der vorliegenden Mitteilung liegen sechs Probeserien zugrunde, die an folgenden Daten erhoben worden sind: 24.-26. Oktober 1967, 11.-13. März 1968, 1.-3. Juli 1968, 21.-23. September 1968, 6.-8. September 1969, 11.-13. Oktober 1970. Untersucht wurden jeweils der Silser See, der Silvaplaner See und der St.Moritzer See an der tiefsten Stelle (Abb.1). Den Champfèrsee, der gleichsam ein Becken des Silvaplaner Sees bildet, konnten wir aus arbeitstechnischen Gründen nicht berücksichtigen.

Die Wasserproben wurden aus folgenden Tiefen entnommen:

a) Silser See: $0 ; 1 ; 2,5 ; 5 ; 7,5 ; 10 ; 12,5 ; 15 ; 20 ; 30 ; 50 ; 65-70 \mathrm{~m}$ (Grundprobe).

b) Silvaplaner See: $0 ; 1 ; 2,5 ; 5 ; 7,5 ; 10 ; 12,5 ; 15 ; 20 ; 30 ; 50 ; 70-75 \mathrm{~m}$ (Grundprobe).

c) St. Moritzer See: $0 ; 1 ; 2,5 ; 5 ; 7,5 ; 10 ; 12,5 ; 15 ; 20 ; 30 ; 39-41 \mathrm{~m}$ (Grundprobe).

Die Wasserproben aus $1 ; 2,5 ; 7,5$ und 12,5 m Tiefe wurden meistens nur für die quantitative Phytoplanktonbestimmung (Biomasse und Photosyntheserate) gebraucht, während die restlichen Proben sowohl chemisch als auch planktologisch 
untersucht wurden. Die physikalisch-chemischen Bestimmungen erfolgten nach den an der Limnologischen Abteilung der Eidg. Anstalt für Wasserversorgung, Abwasserreinigung und Gewässerschutz gebräuchlichen Methoden ${ }^{1}$ ). Das Phytoplankton wurde im Umkehrmikroskop nach Utermöhl quantitativ erfasst und seine Biomasse aufgrund seines Volumens berechnet. Ferner liegen vom Silser und St. Moritzer See je fünf Profile der Photosyntheserate mittels der $\mathrm{C}_{14}$-Methode nach Steeman-Nielsen vor. Für eine erste Bearbeitung des Zooplanktons wurden Netzproben gesammelt,

1) Die genauen Zahlenwerte folgender bei der chemisch-physikalischen Untersuchung berücksichtigten Komponenten sind an der Limnologischen Abteilung der Eidg. Anstalt für Wasserversorgung, Abwasserreinigung und Gewässerschutz archiviert und stehen Interessenten zur Verfügung: Temperatur, Leitfähigkeit, pH, Transmission, Sauerstoff, gelöste Kohlensäure, Karbonathärte, Gesamthärte, Kieselsäure*), Phosphat-, Gesamtphosphor (roh und filtriert), Nitrit-*), Nitrat-Ammonium-*), Kjeldahl-Stickstoff (rolı und filtriert), Eisen*), Mangan*), Chlorid*) und Sulfat*).

*) Diese Komponenten wurden nicht in allen Probeserien bestimmt.

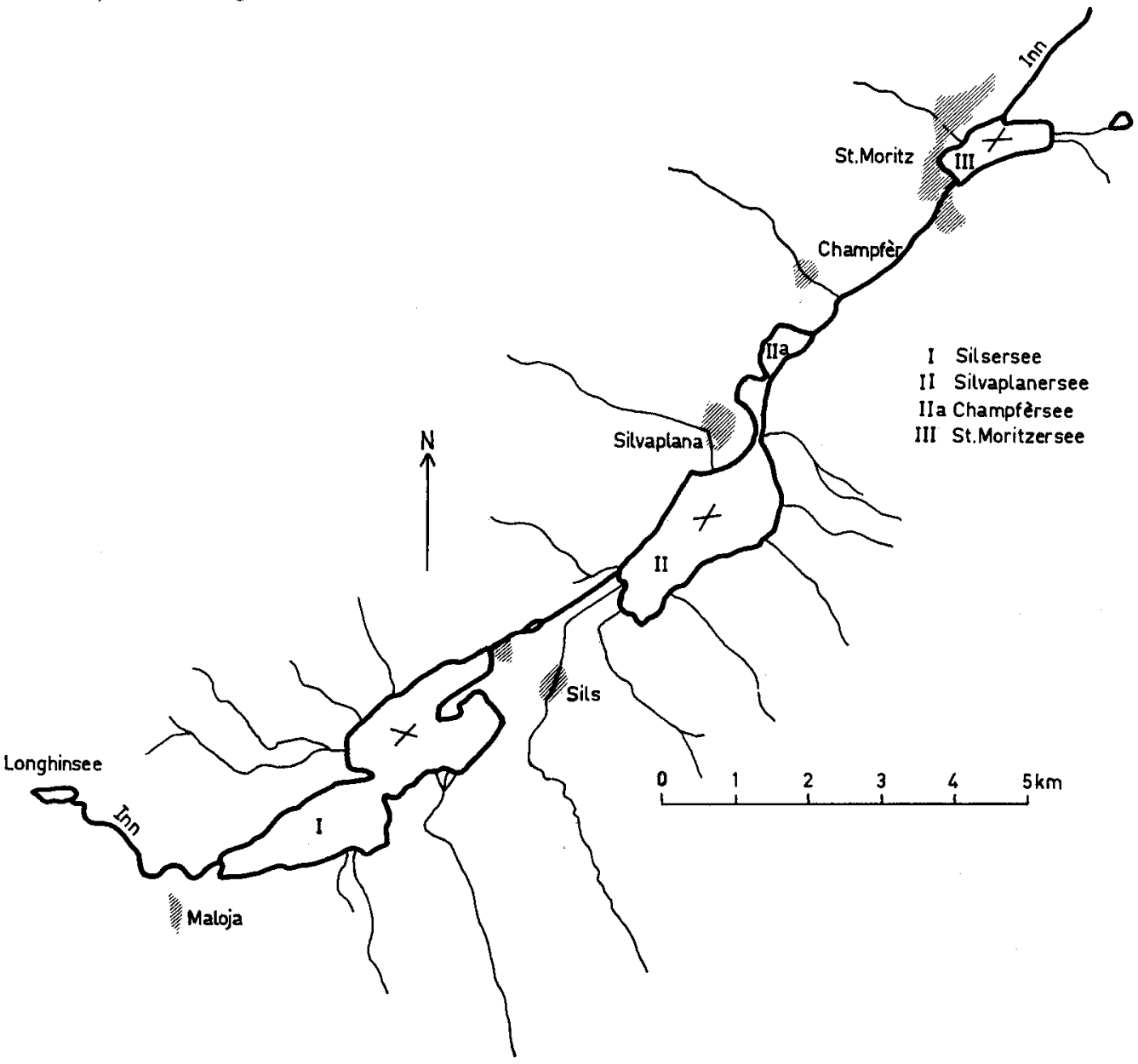

Abb. 1. Lage der Probenahmestellen. 
die jedoch noch nicht verarbeitet worden sind. Bakteriologische Bestimmungen wurden nicht durchgeführt.

Für die Mithilfe und Mitarbeit bei den Labor- und Feldarbeiten möchte ich folgenden Arbeitskollegen aufs herzlichste danken: Herrn dipl. sc. nat. H. Bührer, Herrn H. Bachmann, Herrn Dr. E. Märki, Herrn B. Ribi, Herrn dipl. sc. nat. E. Schegg, Herrn dipl. sc. nat. Szábo, Herrn Dr. U. Zimmermann. Danken möchte ich Herrn C. Raselli, jun., ehemaligem Fischereiaufseher des Oberengadins, und seinem Nachfolger A. Klucker, die uns in entgegenkommender Weise das Motorboot für die Probenahmen auf dem Silser und Silvaplaner See und das Laboratorium der Fischzuchtanstalt St. Moritz für Arbeiten an Ort und Stelle zur Verfügung gestellt haben. Ferner sei der Gemeinde St. Moritz, die uns jeweils das Gemeindeboot für die Probenahmen auf dem St.Moritzer See überliess, gedankt. Für die Messungen der $C_{14}$-Proben habe ich Frau Dr. M. M. Bezzegh, Leiterin der Radiologischen Abteilung an der Eidg. Anstalt für Wasserversorgung, Abwasserreinigung und Gewässerschutz, zu danken. Mein Dank gilt auch Herrn Dr. H. Ambühl, Leiter der Limnologischen Abteilung der Eidg. Anstalt für Wasserversorgung, Abwasserreinigung und Gewässerschutz, für die kritische Durchsicht des Manuskriptes. Er stellte ausserdem Vergleichsdaten von Mittellandseen zur Verfügung.

\section{Lage, Morphologie}

Unter dem Begriff «Engadiner Seen» werden die vier Seen Silser See, Silvaplaner See, Champfèrsee und St. Moritzer See zusammengefasst. Sie liegen im obersten Teil des Inntales, das sich von SW nach NE erstreckt, ganz in der Nähe der heutigen Quelle des Inns, der in $2480 \mathrm{~m}$ über Meer am Longhinsee, oberhalb der Malojapasshöhe (etwa $1800 \mathrm{~m}$ ü.M.), entspringt (Abb.1). Die Felsunterlage der Seebecken und ihrer Einzugsgebiete wird durch die vorwiegend kristallinen Gesteine (wie Granite, Gneisse, Ophiolithe) der hochpenninischen und unterostalpinen Decken gebildet.

Bedeutungsvoll für die Entstehung der Engadiner Seen ist der Umstand, dass offenbar nach dem Rückzug der mächtigen Talgletscher der letzten Eiszeit (Würmeiszeit) in den durch Glazialerosion geschaffenen Vertiefungen des Talbodens mächtige Toteismassen zurückblieben. Beim Schmelzen derselben entstanden zunächst zwei Seen, ein etwa $12 \mathrm{~km}$ langer von Maloja bis Champfèr und ein kleinerer im Becken von St.Moritz. Durch starke Schuttführung der Seitenbäche des Inns wurden Deltas gebildet, die von beiden Seiten in das grosse Becken hineinwuchsen, so dass es geteilt und in die heutigen Seen gegliedert wurde.

In Tab. 1 sind die wichtigsten morphologischen Daten der Seen zusammengestellt. Daraus geht hervor, dass der St. Moritzer See im Vergleich zu den beiden

Tab. 1. Morphologische Daten.

\begin{tabular}{|c|c|c|c|c|}
\hline & & Silser See & Silvaplaner See & St. Moritzer See \\
\hline Spiegelhöhe (m) & $\cdots$ & 1800 & 1794 & 1771 \\
\hline Fläche $\left(\mathrm{km}^{2}\right)$ & . . . & 4,1 & 2,7 & 0,8 \\
\hline Tiefe, max. (m) & . . . . & 71 & 77 & 44 \\
\hline Tiefe, mittlere $(\mathrm{m})$ & . . . & 35 & 48 & 25 \\
\hline Inhalt $\left(\mathrm{km}^{3}\right)$ & . . . & 0,15 & 0,14 & 0,02 \\
\hline Länge $(\mathrm{km}) \cdot$. . & . . & 4,8 & 3,1 & 1,7 \\
\hline Breite $(\mathrm{km}), \ldots$ & . . . & 2,3 & 1,4 & 0,6 \\
\hline
\end{tabular}


anderen Seen, die sich in Grösse und Tiefe ähnlich sind, viel kleiner ist und eine geringere Tiefe aufweist.

\section{Resultate}

Temperatur. Als Beispiele des thermischen Verhaltens der untersuchten Engadiner Seen sind in Abb.2 die Temperaturkurven dargestellt. Zur Zeit der Probenahme im März 1968 lag eine trübe, $70-75 \mathrm{~cm}$ dicke Eisschicht mit etwa $20-50 \mathrm{~cm}$ Schnee auf den Seen. Die Profile lassen deutlich erkennen, in welcher Reihenfolge die Seen zugefroren waren, nämlich zuerst der St.Moritzer See, dann der Silser See und zuletzt der Silvaplaner See, dessen Wassermassen sich bis in $30 \mathrm{~m}$ Tiefe auf weniger als $3^{\circ} \mathrm{C}$ auskühlten, bevor sich eine zusammenhängende Eisdecke bilden konnte. Die Windeinwirkung muss bei diesem wohl am stärksten gewesen sein. Aus den Kurven vom Juli 1968 lesen wir, dass Silser und Silvaplaner See vermutlich von grossen Schmelzwassermengen durchflutet waren, so dass sich im Metalimnion kein steiles Gefälle ausbilden konnte. Der St.Moritzer See hingegen zeigte eine deutliche Sprungschicht, die möglicherweise schon im Frühjahr durch den Wechsel von Schön- und Schlechtwetterperioden bis in $20 \mathrm{~m}$ Tiefe hinuntergedrückt worden war. Im Herbst (September 1968) befanden sich die Seen schon in Teilzirkulation, wobei die Sprungschicht des St.Moritzer Sees im Laufe des Sommers bis $30 \mathrm{~m}$ hinunter verlagert worden war und die eher schwach ausgebildeten Sprungschichten des Silser und Silvaplaner Sees zwischen 15 und $25 \mathrm{~m}$ bzw. 15 und $35 \mathrm{~m}$ Tiefe lagen. Die Temperaturprofile vom Oktober 1967, September 1969 und 1970 zeigen für die einzelnen Seen die erwähnten typischen Merkmale des Temperaturverlaufes in den Herbstmonaten (Abb.2).

Vergleichen wir die Temperaturen der epilimnischen Schichten (Tab.2) miteinander. Alle drei Seen besitzen im Sommer eine relativ niedere Temperatur des Oberflächenwassers. Gegenüber den Mittellandseen liegt sie etwa $10^{\circ} \mathrm{C}$ tiefer. Bedingt wird dieser Unterschied durch die Höhenlage (die Eisschicht besteht rund fünf Monate), durch den Zufluss von Schmelz- und Gletscherwasser und durch die starke Einwirkung des «Malojawindes», der bei stabiler Wetterlage täglich etwa um $11 \mathrm{Uhr}$ vom Bergell (SW-Wind) her zu blasen beginnt und zum Teil starke Wellen auf den Seen erzeugt. Ferner fällt in Tab. 2 auf, dass der Silser See wider Erwarten in fünf Profilen der wärmste ist. Der Temperaturunterschied zwischen Silser und Silvaplaner See variiert stets zwischen 1,0 und $2,4^{\circ} \mathrm{C}$. Im Hypolimnion betragen die Unterschiede der beiden Seen $0,6-0,9^{\circ} \mathrm{C}$ (Grund). Wahrscheinlich wird die tiefere Temperatur des Silvaplaner Sees durch die grössere Zahl an Zuflüssen, die zum Teil kaltes Schmelzwasser führen, bedingt. Diese Aussage kann wohl deshalb gemacht werden, da die Temperaturunterschiede des Silser und Silvaplaner Sees im Epilimnion zur Zeit der Julimessungen, das heisst zur Zeit der Schneeschmelze, am grössten waren $\left(2,4^{\circ} \mathrm{C}\right.$; vgl. Tab.2). Andererseits steht der Silser See möglicherweise unter etwas stärkerem Windeinfluss, so dass durch die grössere Turbulenz auch mehr Kalorien aufgenommen bzw. abgegeben werden. Vergleichen wir nämlich die Temperaturen der Tiefenwasser miteinander (Tab.2), stellen wir fest, dass sich der Silser See in der Tiefe etwas stärker ausgekühlt (März 1968) hatte als der Silvaplaner See, obgleich seine Tiefentemperaturen im Sommer und Herbst (Tab.2) höher sind. 

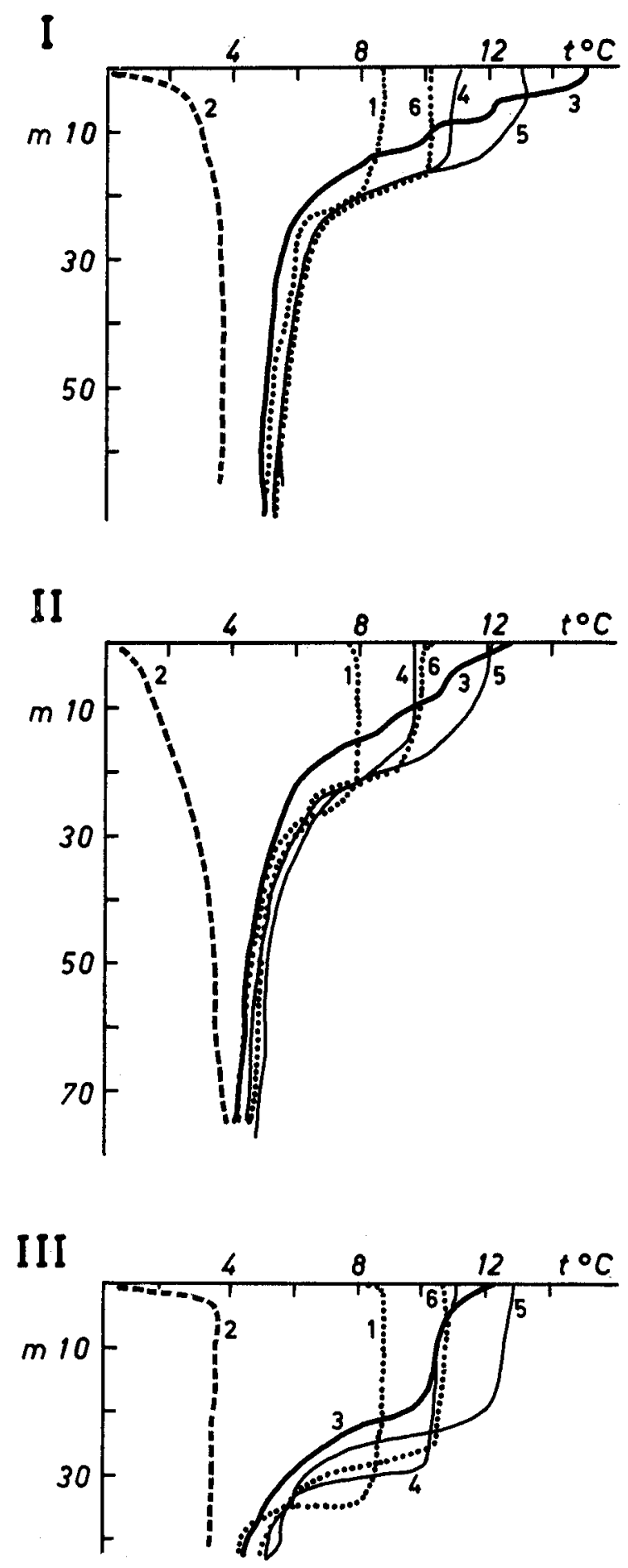

Abb. 2. Temperaturprofile: 1 24.-26. Oktober 1967, 2 11.-12. März 1968, 3 1.-2. Juli 1968, 4 21.-23. September 1968, 5 6.-7. September 1969, 6 11.-13. Oktober 1970. I Silser See, II Silvaplaner See, III St.Moritzer See. 
Tab. 2. Silser, Silvaplaner und St. Moritzer See: Unterschiede der Wassertemperatur $\left(t^{\circ} \mathrm{C}\right)$.

\begin{tabular}{|c|c|c|c|c|c|}
\hline & $\begin{array}{l}\text { Silser } \\
\text { See }\end{array}$ & $\begin{array}{l}\text { Silvaplaner } \\
\text { See }\end{array}$ & $\begin{array}{l}\text { St. Moritzer } \\
\text { See }\end{array}$ & $t^{\circ} \mathrm{C}_{1}$ & $t^{\circ} \mathrm{C}_{2}$ \\
\hline \multicolumn{6}{|l|}{ 24.-26. Oktober 1967} \\
\hline Oberflächenwasser & 8,6 & 7,6 & 8,2 & $+1,0$ & $+0,6$ \\
\hline Tiefenwasser & 5,0 & 4,3 & 4,4 & $+0,7$ & $+0,1$ \\
\hline \multicolumn{6}{|l|}{ 11.-13. März 1968} \\
\hline Oberflächenwasser & _- & - & - & - & - \\
\hline Tiefenwasser & 3,6 & 3,9 & 3,4 & $-0,3$ & $-0,5$ \\
\hline \multicolumn{6}{|l|}{ 1.-3. Juli 1968} \\
\hline Oberflächenwasser & 14,9 & 12,5 & 12,8 & $+2,4$ & $+0,3$ \\
\hline Tiefenwasser & 4,9 & 4,0 & 4,4 & $+0,9$ & $+0,4$ \\
\hline \multicolumn{6}{|c|}{ 21.-23. September 1968} \\
\hline Oberflächenwasser & 10,8 & 9,6 & 10,9 & $+1,2$ & $+1,3$ \\
\hline Tiefenwasser & 5,3 & 4,4 & 5,2 & $+0,9$ & $+0,8$ \\
\hline \multicolumn{6}{|l|}{ 6.-8. September 1969} \\
\hline Oberflächenwasser & 13,0 & 12,0 & 12,8 & $+1,0$ & $+0,8$ \\
\hline Tiefenwasser & 5,5 & 4,6 & 5,0 & $+0,9$ & $+0,4$ \\
\hline \multicolumn{6}{|l|}{ 11. -13 . Oktober 1970} \\
\hline Oberflächenwasser & 10,1 & 9,9 & 10,5 & $+0,2$ & $+0,6$ \\
\hline Tiefenwasser & 5,2 & 4,6 & 4,8 & $+0,6$ & $+0,2$ \\
\hline
\end{tabular}

$\mathrm{t}^{\circ} \mathrm{C}_{1}=$ Temperaturdifferenz $z$ wischen Silser und Silvaplaner See

$\mathrm{t}^{\circ} \mathrm{C}_{2}=$ Temperaturdifferenz $z$ wischen St. Moritzer und Silvaplaner See

Tab. 3. Tiefen $(\mathrm{m})$, in welchen noch $10 \%$ der Oberflächenintensität $(\mathrm{I}=0,05)$ gemessen wurde.

\begin{tabular}{|c|c|c|c|c|c|c|c|c|c|c|}
\hline & \multicolumn{3}{|c|}{ Oktober 1967} & \multicolumn{3}{|c|}{ Juli 1968} & \multicolumn{2}{|c|}{ Sept. 1968} & \multicolumn{2}{|c|}{ Sept. 1969} \\
\hline & I & II & III & I & II & III & I & III & I & III \\
\hline $\mathrm{BG}_{2}$ (blau) & 9 & 9 & 5 & 5 & 6,5 & 5,5 & 8 & 5,5 & 8,5 & 7 \\
\hline $\mathrm{VG}_{9}$ (grün) & 13,5 & 13,5 & 9,5 & 12 & 13 & 7 & 6,5 & 8,5 & 12,5 & 11 \\
\hline $\mathrm{RG}_{2}$ (rot) & 4,5 & 4,5 & 4 & 7 & 6 & 5 & 7 & 4 & 6,5 & 5,5 \\
\hline $\mathbf{I}=$ Silser See & \multicolumn{4}{|c|}{ II $=$ Silvaplaner See } & \multicolumn{4}{|c|}{ III $=$ St. Moritzer See } & & \\
\hline
\end{tabular}

Transmission. Parallel zu den Produktionsmessungen wurde jeweils die Transmission für den blauen $\left(\mathrm{BG}_{12} 417 \mathrm{~nm}\right)$, den grünen $\left.\mathrm{VG}_{9} 525 \mathrm{~nm}\right)$ und den roten $\left(\mathrm{RG}_{2} 652 \mathrm{~nm}\right)$ Wellenbereich gemessen. In Tab. 3 sind die Tiefen, in welchen noch $10 \%$ der Oberflächenintensität vorhanden waren, eingetragen. Bis auf eine Ausnahme (September 1968, Silser See) drang das grüne Licht jeweils am tiefsten ins Wasser ein, was bei einem Grossteil der Seen aus der Literatur der Fall ist [4, 9, 12]. Die grösste Tiefe erreichte es mit 13,5 $\mathrm{m}$ im Silser und Silvaplaner See im Herbst 1967. In den zwei Messreihen, die vom Silvaplaner See vorliegen, verhält er sich optisch fast gleich wie der Silser See. Der St. Moritzer See dagegen zeigt in sozusagen allen Messungen für die drei Spektralbereiche niederere Werte als der Silser See. Vergleiche mit Transmissionswerten anderer Seen anzustellen, ist mit der geringen Anzahl Messungen, die vorliegen, etwas schwierig. Im Vierwaldstätter See, Stelle Horw [4], erreichten die drei Wellenbereiche Blau, Grün und Rot 10\%-Maximalwerte 
von $8,2 \mathrm{~m}$ bzw. 12,3 und 4,5 m, und zwar in der Zeit sehr geringer Produktion (Wintermonate). Diesen Zahlen gegenüber haben wir Maximalwerte von $9 \mathrm{~m}, 13,5 \mathrm{~m}$ und $7 \mathrm{~m}$ gemessen (vgl. Tab. 3). Die Unterschiede sind demnach nicht sehr gross, was auch im Vergleich der Assimilationsraten dieser Seen zum Ausdruck kommt.

Vergleichen wir jedoch unsere Werte mit denen von Pechlaner [8] und RoDHE [10], die an hochalpinen Seen gearbeitet haben, treten die Transmissionswerte der Engadiner Seen stark zurück.

Leitfähigkeit. Die Leitfähigkeit $\varkappa_{\mathbf{2 0}}$ vermittelt eine Vorstellung über den Gesamtsalzgehalt. Sie ist hauptsächlich abhängig von der Gesteinsunterlage; in Kalkgebieten beispielsweise ist sie hoch. Silser und Silvaplaner See zeigten eine relativ geringe Leitfähigkeit mit ungefähr $95 \mu \mathrm{s} / \mathrm{cm}$ bzw. $110 \mu \mathrm{s} / \mathrm{cm}$ im März 1968. Innerhalb der Messprofile wurden nur geringe Unterschiede zwischen Oberflächen- und Tiefenwasser festgestellt. Im St.Moritzer See war die Leitfähigkeit gegenüber den beiden anderen Seen erhöht (ungefähr $165 \mu \mathrm{s} / \mathrm{cm}$ ). Vergleichshalber seien Leitfähigkeitswerte vom Luganer See erwähnt, die sich zur Zeit der Zirkulation im März 1969 zwischen 200 und $230 \mu \mathrm{s} / \mathrm{cm}$ bewegten.

Vor allem aber sind die Unterschiede der Leitfähigkeit zwischen Oberfläche und Tiefe im St. Moritzer See ausgeprägter:

\begin{tabular}{lccc}
\hline & Silser See & Silvaplaner See & St. Moritzer See \\
\hline Leitfähigkeit & & & \\
in $\mu$ s $/ \mathrm{cm}$ & 87 & 95 & 108 \\
minimal & 104 & 122 & 185 \\
maximal & 104 & & \\
\hline
\end{tabular}

In Abb. 3 sind die Leitfähigkeitskurven wiedergegeben. Dabei tritt analog wie bei der Temperatur die Ähnlichkeit von Silser und Silvaplaner See hervor. Ihnen steht der St. Moritzer See gegenüber, der sich von diesen beiden hauptsächlich durch eine deutliche Schichtung in Epi-, Meta- und Hypolimnion unterscheidet.

Sauerstoff. Die Sauerstoffkurven des Silser und Silvaplaner Sees verliefen in der Stagnationszeit (Abb.3) sozusagen gleichmässig mit Werten zwischen 9 und $10 \mathrm{mg} / \mathrm{l}$ in den Oberflächenschichten und zum Teil mit einer geringen, fast unbedeutenden Sauerstoffabnahme im Hypolimnion. Eine maximale Abnahme von $9,19 \mathrm{mg} / \mathrm{l}(0 \mathrm{~m})$ auf 5,56 mg/l (Grund, $75 \mathrm{~m}$ ) zeigte der Silvaplaner See im Oktober 1967, während der Silser See von 9,24 mg/l (0 m) auf 6,74 mg/l (Grund, $68 \mathrm{~m}$ ) gesunken war. Auffallend waren zwei Werte. Im Juli 1968 betrug der Sauerstoffgehalt im Silser See in 5 und $7,5 \mathrm{~m}$ Tiefe rund $12 \mathrm{mg} / \mathrm{l}$. Dieses Maximum wurde kaum durch eine starke Algenentwicklung verursacht (vgl. Photosyntheseraten zu dieser Zeit, Tab.9). Eine Interpretation dieser hohen Werte ist schwierig, da aus dieser Zeit nur ein Profil vorliegt. Vermutlich schichtete sich in dieser Tiefe das Wasser eines Zuflusses ein. Bemerkenswert ist ausserdem die leichte $\mathrm{O}_{2}$-Zunahme in der Tiefe des Silvaplaner Sees im Juli und September 1968 (Abb.1). Wahrscheinlich ist diese Zunahme auf die grössere Löslichkeit des Sauerstoffes bei tieferen Temperaturen zurückzuführen.

Ganz anders liegen die Sauerstoffverhältnisse des St. Moritzer Sees (Abb.3). Der Sauerstoffgehalt nimmt von 20 auf $30 \mathrm{~m}$ Tiefe langsam und von 30 bis $40 \mathrm{~m}$ sehr 

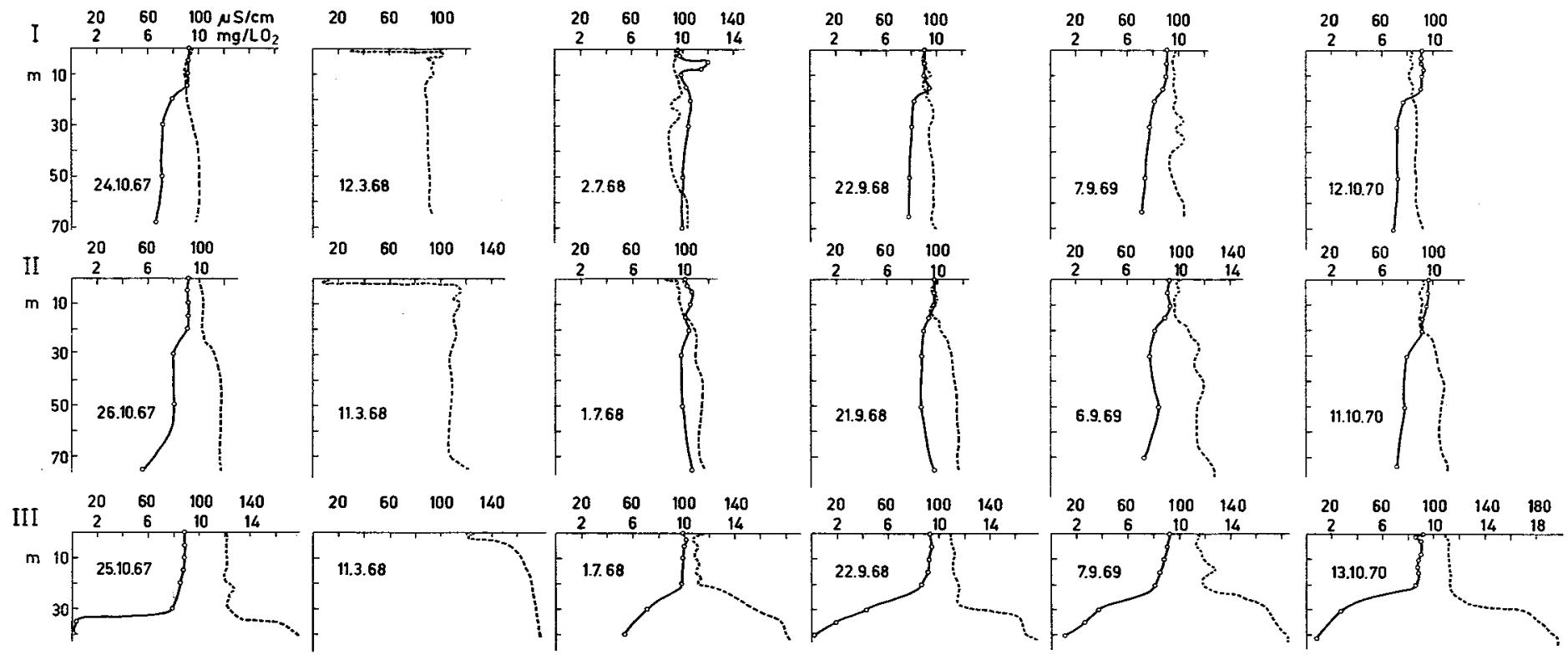

Abb. 3. Sauerstoff $\mathrm{O}_{2}\left(\mathrm{O}_{-O}\right)$ und Leitfähigkeit $\varkappa_{20}(--)$. I Silser See, II Silvaplaner See, III St. Moritzer See. 
schnell ab und erreicht im September 1968 0,18 mg/l (Oktober 1967 0,12 mg/l O , September 1969 1,05 mg/l O , Oktober 1970 0,67 mg/l) über dem Grund. Dieser starke Sauerstoffschwund weist auf eine beträchtliche Belastung des Sees mit organischer Substanz hin. H. Schmassmann [14] und W. und H. Schmassmann [15] haben schon 1918 auf die Belastung des Sees mit Abwasser von St. Moritz hingewiesen. Ihre Sauerstoffprofile vom Oktober 1946 zeigen sehr ähnliche Resultate. Leider konnten die an sich wichtigen $\mathrm{O}_{2}$-Proben vom März 1968 infolge Frost nicht ausgewertet werden.

Bezüglich ihres Sauerstoffgehaltes sind der St.Moritzer See als eutroph, die beiden anderen Seen als oligotroph zu beurteilen.

Karbonathärte. Im Vergleich zu den schweizerischen Mittellandseen weisen die drei Engadiner Seen eine geringe Karbonathärte auf (vgl. Vierwaldstätter See mit 10 und Greifensee mit gut $20^{\circ} \mathrm{fr}$.H.). VollenwEIdER [17] teilt die Seen aufgrund ihrer Alkalinitätsdifferenzen zwischen Winter- und Sommerproben in Trophiegruppen ein. Wie aus Tab. 4 ersichtlich ist, sind die Differenzen für alle drei Seen ausserordentlich klein. Dabei würde man gemäss dem Sauerstoffprofil beim St. Moritzer See eine grössere Differenz erwarten. Was die Alkalinitätsdifferenz anbelangt, müssten alle drei Seen als oligotroph bezeichnet werden.

Phosphor. Am zuverlässigsten kann der Zustand eines Sees anhand des Phosphorgehaltes zur Zeit seiner Winterzirkulation beurteilt werden. So haben wir in Tab.5 den Gehalt an Phosphat-P und Gesamt-P der drei Seen zur Zeit der Märzprobe 1968 zusammengestellt und vergleichshalber dazu Zahlen des mesotrophen Vierwaldstätter Sees (Stelle Kreuztrichter) und des eutrophen Bodensees-Untersees angefügt. Während die Karbonathärtedifferenz bei allen drei Engadiner Seen fast gleich gering ist, kommen in diesen Phosphorkomponenten die Unterschiede zwischen denselben deutlich zum Ausdruck (vgl. auch Abb.4). Silser und Silvaplaner See weisen Werte ähnlich denjenigen des Vierwaldstätter Sees (Stelle Kreuztrichter und Urner See) auf, während der St.Moritzer See mit drei- bis vierfach höherem Gesamt-P und siebenmal grösserem $\mathrm{PO}_{4}$-P-Gehalt dem Untersee nahesteht. Der Gehalt an Gesamt-P ist hoch. Es ist sicherlich zu hoffen, dass diese Werte wieder abnehmen werden, nachdem nun das Abwasser der Gemeinde St. Moritz seit 1968 unterhalb des St. Moritzer

Tab. 4. Karbonathärtedifferenz zwischen Oberflächenproben $(5 \mathrm{~m})$ des Winters und des Sommers.

\begin{tabular}{|c|c|c|c|}
\hline & \multicolumn{2}{|c|}{$\begin{array}{c}\text { Karbonathärte in }{ }^{\circ} \mathrm{fr} . \mathrm{H} . ; \text { in Klammer } \\
\text { (mval/a) }\end{array}$} & $\Delta^{\circ} \mathrm{fr} . \mathrm{H}$ \\
\hline & 11.-13. März 1968 & 21.-23. September 1968 & \\
\hline Silser See & $3,85(0,77)$ & $3,20(0,64)$ & $0,65(0,13)$ \\
\hline Silvaplaner See & $4,50(0,90)$ & $4,10(0,82)$ & $0,40(0,08)$ \\
\hline \multirow[t]{2}{*}{ St. Moritzer See } & $4,60(0,92)$ & $3,90(0,78)$ & $0,70(0,14)$ \\
\hline & 6. März 1962 & 28. August 1962 & \\
\hline \multirow[t]{2}{*}{$\begin{array}{l}\text { Vierwaldstätter See, } \\
\text { Kreuztrichter }\end{array}$} & $10,20(2,04)$ & $7,40(1,48)$ & $2,8 \quad(0,56)$ \\
\hline & 15. März 1962 & 8. August 1963 & \\
\hline Greifensee & $21,8(4,36)$ & $16,6(3,32)$ & $5,2 \quad(1,04)$ \\
\hline
\end{tabular}



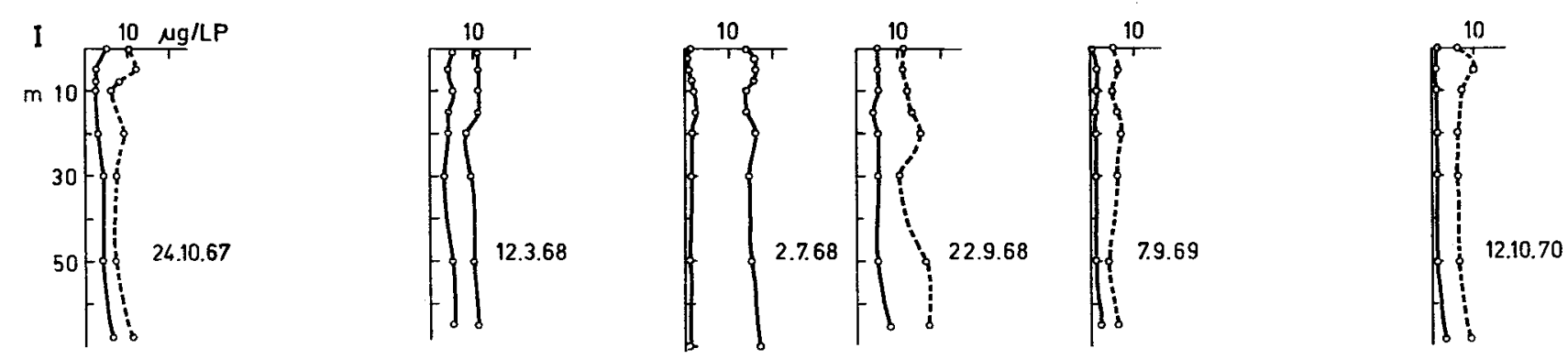

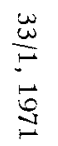
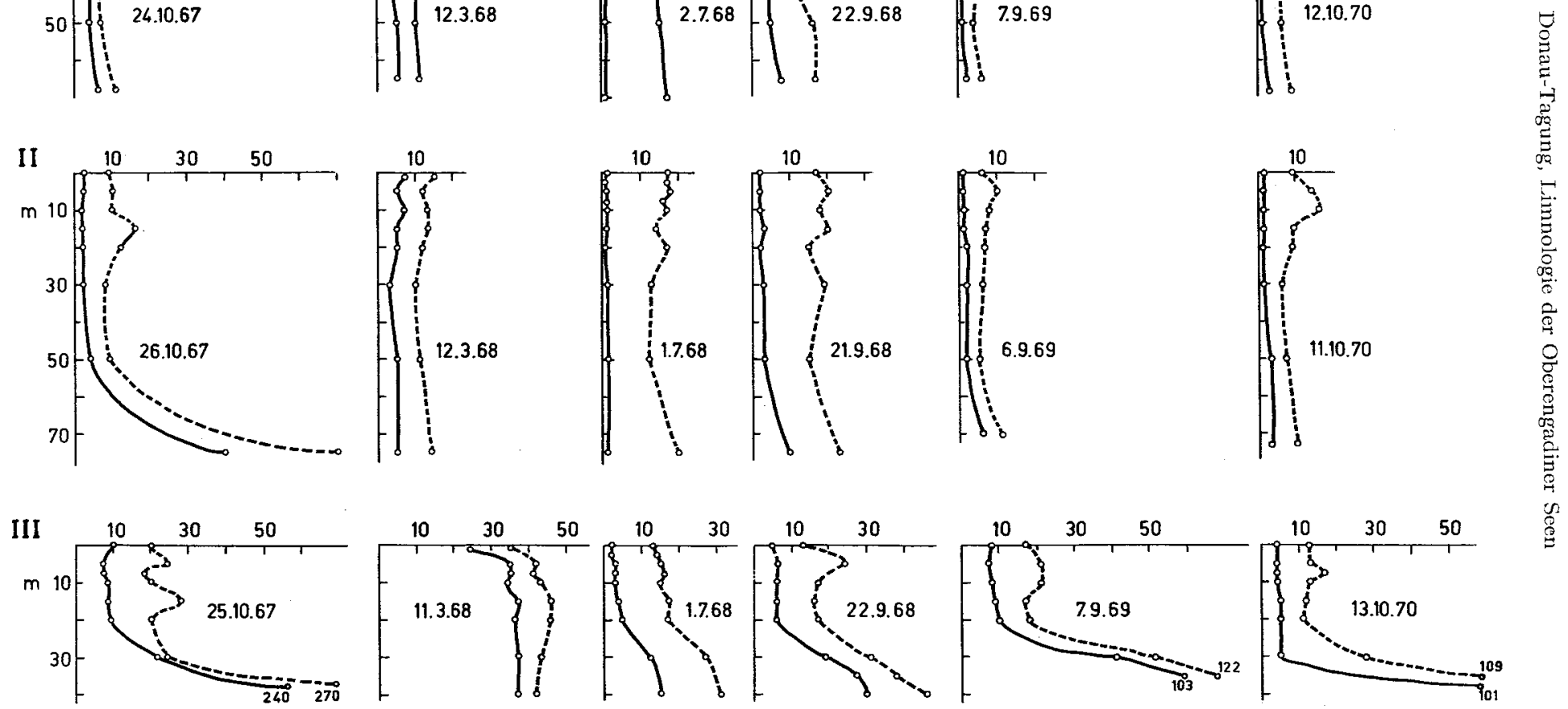

Abb.4. Phosphatphosphor $\mathrm{PO}_{4}-\mathrm{P}\left(\mathrm{O}_{-} \mathrm{O}\right)$ und Gesamtphosphor Ges.-P (O- - O). I Silser See, II Silvaplaner See, III St. Moritzer See, 
Tab. 5. Phosphat-P und Gesamt-P der Engadiner Seen im Vergleich zum Vierwaldstätter See und Bodensee-Untersee zur Zeit der Zirkulation.

\begin{tabular}{lccc}
\hline & & $\begin{array}{c}\mathrm{PO}_{4}-\mathrm{P} \\
\mu \mathrm{g} / \mathrm{l}\end{array}$ & $\begin{array}{c}\text { Gesamt-P } \\
\mu \mathrm{g} / \mathrm{l}\end{array}$ \\
\hline $\begin{array}{l}\text { Silser See } \\
\text { Silvaplaner See }\end{array}$ & 11. März 1968 & 5 & 11 \\
St. Moritzer See & 12. März 1968 & 5 & 13 \\
\hline $\begin{array}{l}\text { Vierwaldstätter See, } \\
\quad \text { Urner See }\end{array}$ & 13. März 1968 & 35 & 43 \\
$\begin{array}{l}\text { Vierwaldstätter See, } \\
\text { Kreuztrichter }\end{array}$ & 10. März 1969 1969 & 6 & 13 \\
\hline Bodensee-Untersee & 17. März 1969 & 4 & 18 \\
\hline
\end{tabular}

Tab. 6. Nitratzehrung im Epilimnion (5 m).

\begin{tabular}{lllr}
\hline & $\begin{array}{l}\mathrm{NO}_{3}-\mathrm{N} \\
\mathrm{mg} / 1\end{array}$ & & $\begin{array}{c}\Delta \mathrm{NO}_{3}-\mathrm{N} \\
\mathrm{mg} / 1\end{array}$ \\
\hline Silser See & $\mathrm{März} \mathrm{1968}$ & September 1968 & \\
Silvaplaner See & 0,16 & 0,08 & 0,08 \\
St. Moritzer See & 0,19 & 0,07 & 0,12 \\
\hline & 0,22 & 0,07 & 0,15 \\
Vierwaldstätter See, & 11. März 1969 & 16. September 1969 & \\
$\quad$ Urner See & 0,43 & 0,17 & 0,26 \\
\hline & 17. März 1969 & 1. September 1969 & \\
Bodensee-Untersee & 0,85 & 0,57 & 0,28 \\
\hline
\end{tabular}

Sees in den Inn geleitet und in Kürze in der regionalen Kläranlage bei Celerina gereinigt wird.

Stickstoff. Zur Zeit der Eisbedeckung (März 1968) war der Gehalt an Nitratstickstoff analog zu den anderen Komponenten fast gleichmässig verteilt (Abb.5). Eine leichte Zehrung hatte in den oberen $5 \mathrm{~m}$ unter Eis schon stattgefunden. Wie $\mathrm{zu}$ erwarten, zeigte der St.Moritzer See mit $0,22 \mathrm{mg} / \mathrm{l}$ Nitratstickstoff die höchste Konzentration, im Vergleich zu Mittellandseen jedoch (Tab.6), wie dem Vierwaldstätter See, Stelle Urner See $(0,43 \mathrm{mg} / 1 \mathrm{~N})$, und dem Bodensee-Untersee $(0,85 \mathrm{mg} / 1)$, eine geringe Konzentration.

Die Nitratzehrung im Epilimnion (vgl. Tab.6) betrug während der Vegetationsperiode im Vergleich zur Zirkulationszeit im St.Moritzer See $0,15 \mathrm{mg} / 1$ und im Silvaplaner und Silser See $0,08 \mathrm{mg} / \mathrm{l}$. Gegenüber dem Vierwaldstätter See (Stelle Urner See) mit $0,26 \mathrm{mg} / \mathrm{l}$ Nitratzehrung und dem Bodensee-Untersee mit $0,28 \mathrm{mg} / \mathrm{l}$ sind diese Werte eher gering. Sie deuten auf eine relativ schwache Algenentwicklung hin.

Der Gesamtstickstoff (Abb.5) erreichte zur Zeit der Eisbedeckung im Silser See gut $0,22 \mathrm{mg} / \mathrm{l}$, im Silvaplaner See 0,28 und im St. Moritzer See 0,33 mg/l. Die Unter- 


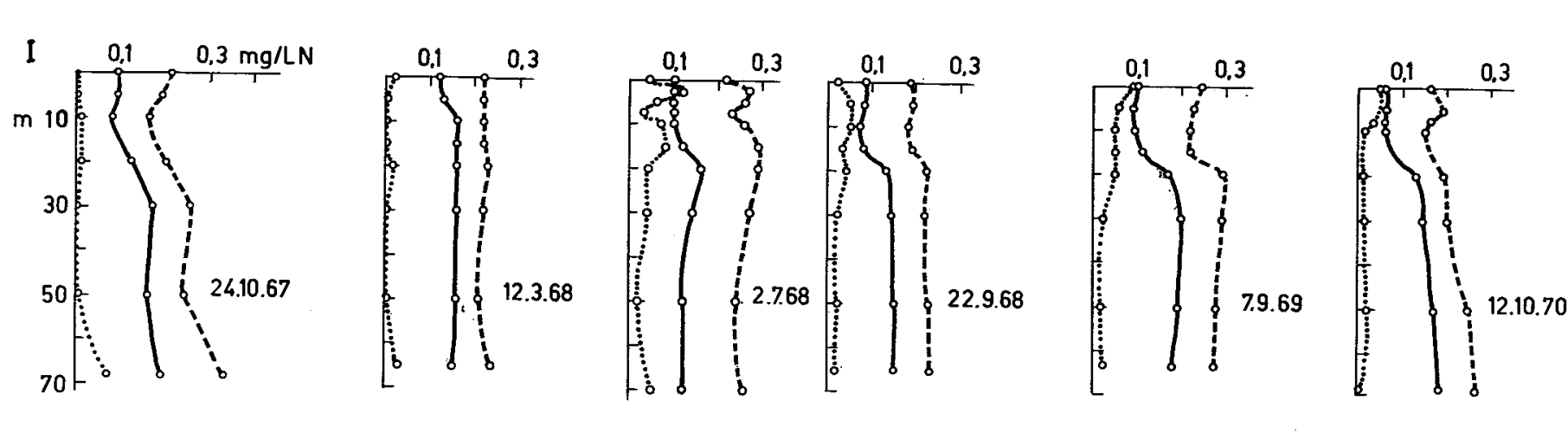

$$
\begin{aligned}
& \text { "y } \\
& \text { on: }
\end{aligned}
$$


schiede sind demnach, wenn auch nicht sehr gross, doch vorhanden. Auch in den Analysenwerten vom Juli 1968 fanden wir in allen drei Seen ähnliche Gehalte an Gesamtstickstoff. Ein eindeutiger Unterschied tritt in den Herbstprofilen (Abb.5) hervor, wobei in den Proben über dem Grund im St. Moritzer See Werte von 0,55 bis $0,67 \mathrm{mg} / 1$ vorlagen, im Silser und Silvaplaner See solche von 0,23 bis $0,33 \mathrm{mg} / 1$ bzw. 0,28 bis $0,41 \mathrm{mg} / \mathrm{l}$ Gesamt-N nachgewiesen wurden.

Den partikulär gebundenen Stickstoff haben wir in Abb.5 eingetragen. Er gibt im grossen ganzen den Verlauf der gebildeten Biomasse wieder. Das Verhältnis Biomasse (B): partikulärem Stickstoff (p. N) variiert stärker als in den Schöpfproben des Vierwaldstätter Sees [7]. So bewegen sich zum Beispiel die Verhältniszahlen B/p.N der Proben unter dem Eis (März 1968) vor allem im Silvaplaner und St. Moritzer See um 1:3; d.h. der organisch gebundene Stickstoff machte etwa 30\% der berechneten Biomasse aus (in den Proben vom Vierwaldstätter See nur rund 5\%). Ähnliche Verhältniszahlen (um 1:3) lagen im September 1968 und im Oktober 1970 im Silser See vor. Diese Zahlen aufgrund weniger Proben zu interpretieren, ist etwas gewagt. Möglicherweise deuten sie auf eine verhältnismässig grosse Menge an organischem Detritus hin. Diese würde im September 1968 mit der starken Trübung zusammenhängen, die vermutlich durch Schnee- und Regenfälle bedingt war. Unter der Eisdecke vom März 1968 waren wahrscheinlich viele Organismen abgestorben, ohne aber vollständig abgebaut gewesen zu sein.

Phytoplankton. Wenn chemische Analysendaten relativ weniger Wasserproben einen See schon recht gut charakterisieren, so sind stichfeste Aussagen aufgrund von Phytoplanktonuntersuchungen erst nach zahlreichen Analysen von lebendem und fixiertem Plankton möglich. Die sechs Planktonprofile der Seen umfassen nur einen Bruchteil eines gründlichen Untersuchungsprogrammes.

Die qualitative Zusammensetzung des Phytoplanktons der sechs Probeserien ist in Tab. 7 zusammengestellt. Bis heute wurden nur fixierte Schöpfproben (mit JKJLösung) analysiert, wobei einige Formen, speziell Flagellaten, aber auch kleine, kugelige Formen ( $\mu$-Algen) nicht eindeutig bestimmt werden konnten. Rund 40 Phytoplankter wurden beobachtet. Wir sind überzeugt, dass die Anzahl Arten bei eingehender Untersuchung (zeitlich dichtere Probenahme und Analyse lebenden Materials) beträchtlich erhöht würde.

Auffallend ist das Fehlen der Blaualgen, auch im nährstoffreicheren St. Moritzer See. Dies hängt wahrscheinlich mit der Lage (Höhe über Meer und Einzugsgebiet von Gletschern) und den damit verbundenen tiefen Sommertemperaturen im Epilimnion zusammen. Die Grünalgen waren mit neun Arten, die Chrysophyceen mit sieben, die Kieselalgen mit zehn, die Cryptophyceen und Peridineen mit vier bzw. drei Arten vertreten. Die Seen zeigten in qualitativer Hinsicht kaum Unterschiede. Vergleicht man die festgestellten Formen mit denen der Mittellandseen, so sind in diesen Probeserien drei neue Arten (die aber in fixiertem Zustand nicht bestimmt werden konnten), nämlich ein Chlamydomonas, eine Chromulina und ein Gymnodinium beobachtet worden.

Die quantitative Bestimmung des Phytoplanktons (Tab. 8) ergab, dass die Chlorophyten in allen Seen nur einen kleinen Teil der Biomasse ausmachten. Oft erreichten sie keine 0,1\%. Der höchste Anteil betrug im Oktober 1967 16,2\% bzw. im Oktober 
Tab. 7. Qualitative Zusammensetzung des Phytoplanktons aufgrund fixierter Proben (JKJ-Lösung).

Chlorophyta

Chlamydomonas sp.

Pandorina morum (Müller) Bory

Sphaerocystis schroeteri Chod.

Characium gracilipes F. D. Lambert

Elakatothrix gelatinosa Wille

Cosmarium minimum W. et G. S. West

Staurastrum Brebisonii Archer

Mougeotia sp.

Chrysophyceae

Pseudopedinella erkensis Skuja

Chromulina sp. *)

Erkenia subaequiciliata Skuja

Pseudokephyrion entzii Conrad

Diceras chodati Reverdin

Dinobryon divergens Imhof

Mallomonas sp.

Diatomeae

Cyclotella operculata (AG.) Kg.*)

Stephanodiscus hantzschii Grun.

Stephanodiscus alpinus Hustedt

Stephanodiscus astraea (E.) Grun.

Tabellaria fenestrata (Lyngb.) Kg.*)

Diatoma elongatum (Lyngb.) $\mathrm{Kg}$.

Fragilaria crotonensis Kitton

Asterionella formosa Hasall *)

Synedra acus Kützing

Synedra sp.

Cryptophyceae

Rhodomonas lacustris Pascher et Ruttner*)

Cryptomonas erosa Ehrnb.

Cryptomonas ovata Ehrnb.

Katablepharis ovalis Skuja

Peridineae

Gymnodinium lantzschii Utermöhl

Gymnodinium helveticum Penard

Gymnodinium sp.

*) Massenformen
Silser

See

Silvaplaner

See

St. Moritzer

See

$\begin{array}{ll}+ & + \\ + & + \\ + & + \\ + & + \\ + & +\end{array}$

$\begin{array}{ll}+ & + \\ + & + \\ + & + \\ + & + \\ + & + \\ + & + \\ & +\end{array}$

+
+
+
+
+

$+$

$+$

$+$

$+\quad+$

$\begin{array}{lll}+ & + & + \\ + & + & + \\ + & + & + \\ + & + & + \\ + & + & + \\ + & + & + \\ + & + & + \\ + & + & + \\ + & + & + \\ + & + & +\end{array}$

$\begin{array}{lll}+ & + & + \\ + & + & + \\ + & + & + \\ + & + & +\end{array}$

$\begin{array}{lll}+ & + & + \\ + & + & + \\ + & + & +\end{array}$

$19707,3 \%$ (Silser See). Bedingt waren diese Prozentanteile durch das Vorhandensein von $\mu$-Algen, die sich in diesem Monat bis zu 3,2 Mio. bzw. 1,6 Mio. Zellen/l entwickelt hatten. Als auffallend ist das Auftreten von Mougeotia im St. Moritzer See im September 1969 und Oktober 1970 zu erwähnen, im Silser und Silvaplaner See wurde sie hingegen in keiner Probeserie festgestellt. Mongeotia sp. wurde in den 
Flachlandseen nur in stärker eutrophierten Typen wie Bodensee-Untersee, Zürichsee, Hallwiler See und Vierwaldstätter See in den letzten Jahren gefunden.

Die Chrysophyceen waren im Silser See prozentual stärker vertreten als in den beiden anderen Seen. Mit 50,8\% machten sie im September 1969 sogar mehr als die Hälfte der Biomasse aus. Dieser hohe Anteil ist hauptsächlich auf einen in fixiertem Zustand leider unbestimmbaren Chrysoflagellaten (vermutlich eine Chromulina sp.) zurückzuführen, der in bis zu 1,7 Mio. Ind./1 vorhanden war. Im Vergleich zu den Flachlandseen waren auch Erkenia subaequiciliata, Pseudopedinella erkensis, Diceras chodati, Pseudokephyrion entzii, Uroglena americana und Dinobryon divergens vertreten. Unter diesen hatte Erkenia subaequiciliata und Uroglena americana zur Zeit der Probeserien die grössten Zellzahlen ausgebildet (bis 780000 Zellen/l im Silser See, Oktober 1967).

Die Gruppe der Kieselalgen war im Juli 1968 in allen Seen quantitativ am stärksten entwickelt. Zur Zeit der Probenahme war der "standing crop» in allen drei Seen am höchsten (vgl. Tab. 8 und 9). Im Silser See waren es hauptsächlich Asterionella formosa mit maximal 800000 Zellen $/ 1$ in $2,5 \mathrm{~m}$ Tiefe und Cyclotella operculata mit 9,7 Mio. Zellen/1 in $5 \mathrm{~m}$ Tiefe. Im Silvaplaner See dominierten Asterionella formosa mit 1,5 Mio. Zellen/1 in $5 \mathrm{~m}$ Tiefe und Cyclotella operculata mit 3 Mio. Zellen/1 in $10 \mathrm{~m}$. Ausserdem aber waren im Gegensatz zum Silser See noch Tabellaria fenestrata, Fragilaria crotonensis und Stephanodiscus hantzschii zahlreich vorhanden. Das Kieselalgenplankton des St. Moritzer Sees setzte sich ähnlich wie im Silvaplaner See, mit Asterionella formosa (4,7 Mio. Zellen/1 maximal in 2,5 m Tiefe) und Cyclotella operculata (1,8 Mio. Zellen/l in $5 \mathrm{~m}$ Tiefe), zusammen. Ferner war Tabellaria fenestrata mit 160000 Zellen/l in $10 \mathrm{~m}$ und Fragilaria crotonensis mit 180000 Zellen/l in $5 \mathrm{~m}$ Tiefe vorhanden, sodann eine kleine Synedra sp. mit 550000 Zellen/1 in $5 \mathrm{~m}$ und Stephanodiscus hantzschii mit 620000 Zellen/l in 7,5 m. Während im Silvaplaner und St.Moritzer See die Kieselalgen noch in je zwei bzw. drei weiteren Probeserien (vgl. Tab.8) dominierten, waren es im Silser See hauptsächlich die Gruppen der Cryptophyceen und Chrysophyceen, die einen wesentlichen Anteil an der Biomasse ausmachten.

Die Cryptophyceen setzten sich in allen drei Seen aus den weit verbreiteten Rhodomonas lacustris und $R$. lens und Cryptomonas erosa zusammen. Die höchste Biomasse erreichten sie im Oktober 1967 und September 1969 im St.Moritzer See mit je $1,2 \mathrm{mg} / 1$ in $5 \mathrm{~m}$ bzw. $0 \mathrm{~m}$ Tiefe, bedingt durch die Entwicklung von Cryptomonas. Die höchste Individuenzahl von Rhodomonas wurde im Silser See beobachtet mit 1,6 Mio. Ind./l in $10 \mathrm{~m}$ (7. September 1969), während im Silvaplaner See 1,3 Mio. Ind./1 in 0-10 m am 11. Oktober 1970 vorhanden waren und im St.Moritzer See 950000 Ind./1 in $5 \mathrm{~m}$ im September 1968. Cryptomonas erreichte im St. Moritzer See die höchste Individuenzahl mit 540000 Ind./1 im Oktober 1967, in $5 \mathrm{~m}$ Tiefe.

Die Peridineen waren zur Zeit der Probenahmen in unwesentlicher Zahl vertreten.

Summieren wir die Menge an Biomasse, die jeweils pro Probeserie in einer Wassersäule von 0 bis $20 \mathrm{~m}$ über einem $\mathrm{m}^{2}$ gefunden worden war, und vergleichen wir die Summen der einzelnen Seen miteinander, erhalten wir ein Verhältnis von

Biomasse (Silser See): B (Silvaplaner See) : B (St. Moritzer See) = 1:1,6:2,2.

In anderen Worten: Der St. Moritzer See hatte zur Zeit der Probenahmen die doppelte Menge an Phytoplankton ausgebildet. Diese Zahlen sind natürlich nur mit 
Tab. 8. Prozentuale Biomasseanteile der Algengruppen und durchschnittliche Phytoplanktonbiomasse von 0 bis $20 \mathrm{~m}$ (mg/l).

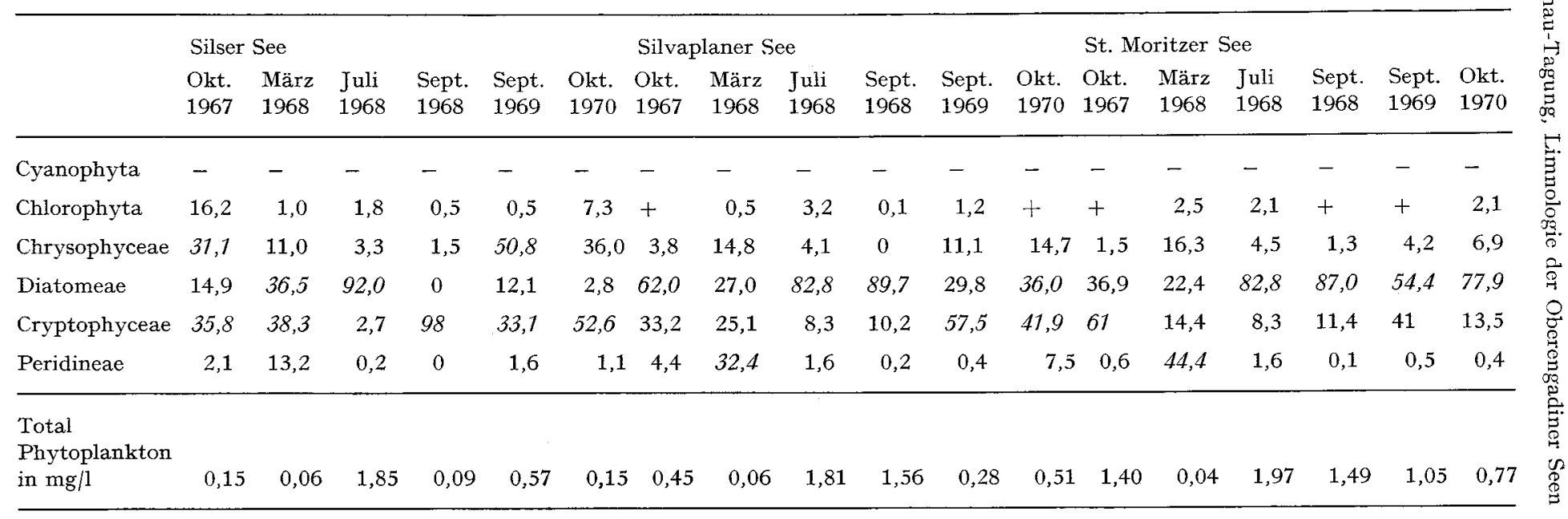


Vorbehalt zu betrachten, da die Anzahl der Probeserien zu klein ist, um sichere Aussagen zu machen. Vergleicht man ferner die quantitative vertikale Verteilung der drei Seen in chronologischer Abfolge, fällt auf, dass die Biomasse im Silser See bald kleinere, bald grössere Mengen erreichte. Besonders eindrücklich war die Abnahme der Biomasse im September 1968. Diese Schwankungen sind vermutlich bedingt durch die Zehrung des Phosphat-Phosphors. Möglicherweise kann aber auch eine starke Trübung von Schmelzwasser die Verminderung der Planktonpopulation verursachen. Auch im Silvaplaner See waren Schwankungen in der Biomasse zu beobachten, während der St.Moritzer See eher regelmässig gleiche Mengen an Biomasse entwickelt hatte (vgl. Tab.8).

Um die Dynamik des Phytoplanktons einigermassen zu erfassen, wurden im Silser See und im St.Moritzer See jeweils $\mathrm{C}_{14}$-Bestimmungen durchgeführt (ausser unter dem Eis im März 1968). Die Daten sind in Tab.9 zusammengestellt.

Im Oktober 1967 und September 1969 wiesen die Assimilationskurven des St.Moritzer Sees deutliche Produktionsmaxima auf (vgl. Abb.6), was nach der Einteilung von FindenegG [3] eher ein Merkmal eutropher Seen ist. Die Assimilationsraten waren jedoch in beiden Seen im Vergleich zu anderen Seen (vgl. RoDHE [9]) gering und erreichten in den je fünf Messdaten nie $100 \mathrm{mg} \mathrm{C}$ ass $/ \mathrm{m}^{2} \mathrm{Tag}$ ), so dass beide Seen bezüglich $\mathrm{C}_{14}$-Assimilation zu den oligotrophen Seen gerechnet werden könnten. Auffallend sind die ausserordentlich tiefen Werte des Silser Sees im September 1968. Diese Werte stimmen mit der niedrigen Biomasse zu jenem Zeitpunkt überein (Tab.9). Die Sichttiefe jedoch war zur Zeit nicht auffallend gross $(6,8 \mathrm{~m})$. Ferner wichen die Transmissionswerte (vgl. Tab.3) im Vergleich zu den anderen Malen etwas $a b$, indem nicht der grüne, sondern der rote Wellenbereich die grösste Tiefe erreichte. Die Planktonproben zeigten starke Trübungen von mineralischen Teilchen, die möglicherweise von starken Niederschlägen herrührten. Diese Trübung erklärt vielleicht auch die geringe Biomasse und die äusserst niedrige Assimilationsrate im Silser See im September 1968.

\section{Schlussfolgerungen}

Aus den Analysendaten der je sechs Probeserien können wir die drei untersuchten Engadiner Seen Silser, Silvaplaner und St. Moritzer See zusammenfassend wie folgt beurteilen:

Silser und Silvaplaner See weisen ähnliche morphologische, chemisch-physikalische und biologische Merkmale auf, wobei die Werte der chemischen Komponenten und der Biomasse des Silvaplaner Sees im allgemeinen etwas höher lagen als diejenigen des Silser Sees.

Silser und Silvaplaner See können in bezug auf den Gehalt an Sauerstoff, Phosphat- und Gesamtphosphor und an Nitrat- und Gesamtstickstoff als oligotroph betrachtet werden. Sie gehören wohl zu den saubersten Seen der Schweiz. Bedingt durch den Fremdenverkehr nimmt die Zahl der Bewohner an diesen Seen zu. Um den heutigen Zustand dieser beiden Seen zu erhalten, ist es dringend notwendig, dass jedes Abwasser von den Seen ferngehalten wird.

Der St.Moritzer See unterscheidet sich von den beiden ersteren Seen, sowohl in Morphologie, Thermik, Chemie als auch in den Biomassewerten, in der Grösse der Kohlenstoffassimilation und z.T. in der Zusammensetzung des Phytoplanktons. 

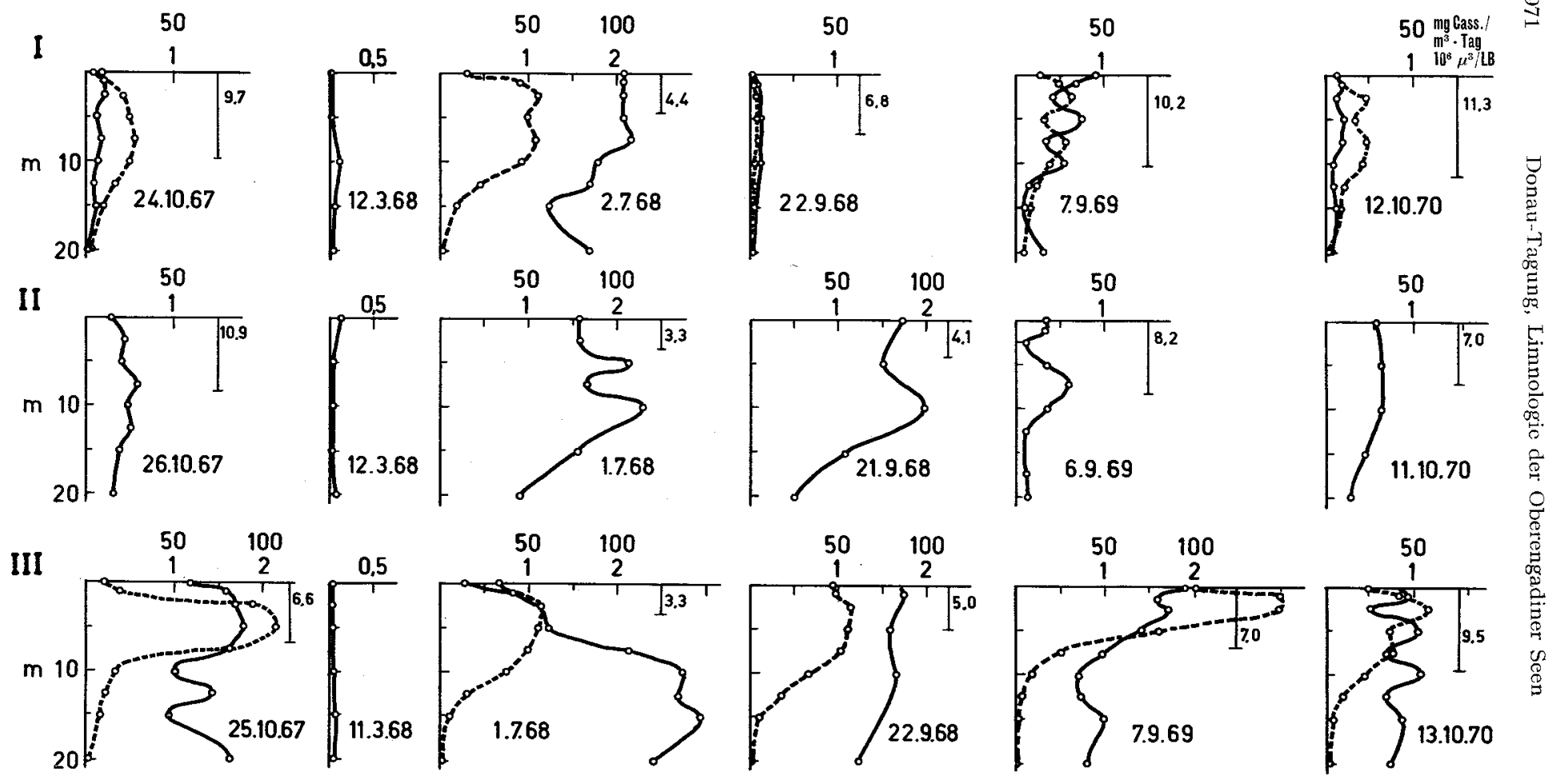

Abb.6. Biomasse B (O-O) und Photosyntheseraten $\mathrm{C}_{\text {ass }}(\mathrm{O}-\mathrm{-}$ ) . Ferner ist die Sichttiefe (m) eingetragen. I Silser See, II Silvaplaner See, III St. Moritzer See. 
Tab. 9. Photosyntheseraten $\left(\mathrm{mg} \mathrm{C}_{\mathrm{ass}} / \mathrm{m}^{3} \cdot \mathrm{h}\right)$, Biomasse $(0-20 \mathrm{~m})$ und Aktivitätskoeffizient. $I=$ Silser See, III $=$ St. Moritzer See.

\begin{tabular}{|c|c|c|c|c|c|c|c|c|c|c|}
\hline \multirow{2}{*}{$\begin{array}{l}\text { Tiefe } \\
\mathrm{m}\end{array}$} & \multicolumn{2}{|c|}{ 24./25. Okt. 1967} & \multicolumn{2}{|c|}{ 1./2. Juli 1968} & \multicolumn{2}{|c|}{ 22./23. Sept. 1968} & \multicolumn{2}{|c|}{ 7./8. Sept. 1969} & \multicolumn{2}{|c|}{ 12./13. Okt. 1970} \\
\hline & I & III & $\mathrm{I}$ & III & $\mathbf{I}$ & III & $\mathrm{I}$ & III & I & III \\
\hline 0,0 & 0,39 & 0,96 & 1,56 & 1,38 & 0,02 & 3,41 & 1,43 & 10,08 & - & - \\
\hline 1,0 & 0,87 & 2,11 & 4,52 & 4,11 & 0,03 & 4,73 & 2,55 & 14,82 & 0,85 & 4,09 \\
\hline 2,5 & 2,12 & 9,39 & 5,51 & 5,70 & 0,04 & 5,66 & 3,14 & 14,70 & 2,26 & 5,79 \\
\hline 5,0 & 2,48 & 10,71 & 4,91 & 5,62 & 0,05 & 5,52 & 1,65 & 8,08 & 1,68 & 3,63 \\
\hline 7,5 & 2,78 & 8,15 & 5,33 & 5,03 & 0,06 & 5,10 & 2,82 & 2,45 & 2,44 & 3,73 \\
\hline 10,0 & 2,46 & 1,57 & 4,61 & 3,72 & 0,04 & 3,23 & 1,91 & 0,88 & 2,11 & 2,07 \\
\hline 12,5 & 1,69 & 1,17 & 2,29 & 1,45 & 0,04 & 1,71 & 1,21 & 0,36 & 1,06 & 0,87 \\
\hline 15,0 & 1,03 & 0,79 & 0,95 & 0,50 & 0,03 & 0,42 & 0,88 & 0,17 & 0,86 & 0,63 \\
\hline 20,0 & 0,27 & 0,03 & 0,18 & 0,08 & 0,00 & 0,01 & 0,52 & 0,03 & 0,28 & 0,09 \\
\hline $\mathrm{mg} \mathrm{C}_{\mathrm{ass}} \mathrm{m}^{2} \cdot \mathrm{h}$ & 33,58 & 79,05 & 64,72 & 57,97 & 0,71 & 58,8 & 33,36 & 83,01 & 27,73 & 43,90 \\
\hline $\mathrm{mg} \mathrm{C}_{\text {ass }} / \mathrm{m}^{2} \cdot \mathrm{Tag}$ & 336 & 790 & 647 & 580 & 7,1 & 590 & 334 & 830 & 277 & 439 \\
\hline mg Biomasse $/ \mathrm{m}^{2}$ & 2730 & 25600 & 35630 & 42870 & 2050 & 31090 & 14230 & 15465 & 2810 & 16050 \\
\hline $\begin{array}{l}\text { Aktivitäts- } \\
\text { koeffizient }\end{array}$ & 0,12 & 0,03 & 0,02 & 0,01 & 0,003 & 0,02 & 0,02 & 0,05 & 0,10 & 0,03 \\
\hline
\end{tabular}


Der ausgeprägte Sauerstoffschwund im Hypolimnion, der hohe Phosphat- und Gesamtphosphorgehalt zur Zirkulationszeit und die Anreicherung dieser Stoffe im Hypolimnion zur Stagnationszeit zeigen den eutrophen Charakter des St.Moritzer Sees an. Dieser Zustand ist offensichtlich die Folge der direkten Einleitung der häuslichen Abwässer von St.Moritz bis zum Jahre 1968. Die Werte der Nitratzehrung im Epilimnion waren im Vergleich zum Silser und Silvaplaner See wohl doppelt so hoch, lagen aber gegenüber Zahlen von eutrophen Seen des Mittellandes eher etwas tiefer, so dass man ihn bezüglich Nitrat- und Gesamtstickstoff kaum als eutroph bezeichnen kann.

Die Seen unterscheiden sich zum Teil auch in der quantitativen Zusammensetzung des Phytoplanktons. Erwähnenswert ist, dass im Silser See nur Asterionella formosa und Cyclotella operculata in grosser Masse beobachtet wurden. Fragilaria crotonensis war nie zahlreich entwickelt gewesen, und Tabellaria fenestrata wurde überhaupt nur in einer Grundprobe festgestellt. Im Silvaplaner und St. Moritzer See hingegen waren Asterionella formosa, Fragilaria crotonensis und Tabellaria fenestrata in allen Probeserien vorhanden gewesen. Fragilaria crotonensis und Tabellaria fenestrata wurden dreimal zur Zeit einer starken Entfaltung erfasst. Wie die Untersuchungen an anderen Seen (z.B. Zürichsee, Vierwaldstätter See) gezeigt haben, deuten Massenentfaltungen von Tabellaria fenestrata auf eine beginnende Eutrophierung hin. SARACENI [13] kam aufgrund von Kulturversuchen zum Schluss, dass die drei Kieselalgen Asterionella, Fragilaria, Tabellaria mit steigendem Trophiegrad eines Sees nacheinander auftreten können. Die massenhafte Entwicklung von Tabellaria fenestrata im Silvaplaner See ist daher um so erstaunlicher, da die Analyse der gelösten Stoffe auf einen oligotrophen Charakter dieses Sees hinweisen. Im St. Moritzer See dagegen ergibt die starke Entfaltung von Tabellaria fenestrata ein Bild, das wie zum Teil die chemischen Komponenten eine stärkere Eutrophierung dieses Sees anzeigt.

Die Biomasse des Phytoplanktons und die Assimilationsraten von fünf Probeserien waren im St.Moritzer See doppelt so gross als im Silser See. Sie lagen jedoch tiefer als in eutrophen Seen des Mittellandes, so dass der St. Moritzer See bezüglich dieser Parameter wiederum eher zu den oligotrophen Seen gerechnet werden könnte. Allerdings war die vertikale Verteilung der Kohlenstoffassimilation in zwei Serien durch ein ausgeprägtes Maximum in den oberen Schichten charakterisiert, was nach der Einteilung von FINDENEGG [3] vor allem in eutrophen Seen der Fall ist.

Aufgrund der Beurteilung der einzelnen chemischen und biologischen Komponenten können der Silser und Silvaplaner See noch als oligotroph betrachtet werden. Der St.Moritzer See zeigt zum. Teil eindeutig eutrophe Merkmale (Sauerstoffschwund, hoher Phosphat- und Gesamtphosphorgehalt, vertikale Verteilung der Assimilationsrate), während das Verhalten anderer Komponenten (Nitrat- und Gesamtstickstoff, Karbonathärte) aufgrund der sechs Probenahmeserien keine eindeutige Aussage über den Trophiegrad dieses Sees erlauben.

\section{Offene Probleme}

Die Resultate der sechs sporadischen Untersuchungsprofile der drei Engadiner Seen Silser, Silvaplaner und St.Moritzer See geben wohl ein Bild über deren chemischen und zum Teil biologischen Zustand und vermitteln Anhaltspunkte über 
deren charakteristische Unterschiede. Doch bilden diese Daten nur Bruchstücke einer einigermassen vollständigen, limnologischen Untersuchung dieser drei Bergseen. Sicher würden eingehendere Forschungsarbeiten wertvolle Vergleiche mit den Ergebnissen von Flachlandseen ermöglichen. Beim Verarbeiten des Probematerials und der Analysendaten sind im wesentlichen folgende Fragen aufgetaucht:

1. Wie ändern die chemischen und physikalischen Komponenten der einzelnen Seen im Laufe des Jahres? (Die Proben sollten in kleinen und regelmässigen Zeitabständen erhoben werden.)

2. Welche Mengen an gelösten Stoffen gelangen durch die Zuflüsse in die einzelnen Seen und wie viele werden in den Becken zurückgehalten?

3. Ist der Gehalt an Nährstoffen in gewissen Zuflüssen stark saisonabhängig (Fremdenverkehr) und wirkt sich derselbe jeweils auf die Bioaktivität der Seen aus? Theoretisch würde man erwarten, dass schon eine relativ kleine zusätzliche Düngung der Seen sich auf die Biologie auswirkt, besonders angesichts der Minimumsfunktion des Phosphors.

4. Welche Mengen an Stickstoff und Phosphor werden aus dem Einzugsgebiet ausgewaschen und abgeschwemmt? (Der grösste Teil ist unbearbeitetes Naturland, Wald und Weidegebiet.)

5. Wie stark ist die Durchflutung der einzelnen Becken?

6. Wird sich der Zustand des St. Moritzer Sees spürbar ändern, nachdem nun kein Abwasser mehr von der Gemeinde St. Moritz in den See gelangt? Oder hängt der hohe Phosphorgehalt und der starke Sauerstoffschwund im Hypolimnion mit - möglicherweise sogar unterseeischen - Mineralquellen zusammen ?

7. Welchen Einfluss haben die Trübstoffe der Schmelzwässer im Frühjahr und Sommer auf Chemismus und Biologie der Seen?

8. Die qualitative und quantitative Entwicklung des Phyto- und Zooplanktons sollte in kurzen Zeitabständen im Laufe wenigstens eines Jahres gründlich verfolgt werden. Welche Faktoren, klimatische, chemische und biotische, beeinflussen die Zusammensetzung der Planktongesellschaften? Welche Arten sind charakteristisch für diese Bergseen?

9. In welcher Tiefe ist in den einzelnen Seen noch eine positive Assimilation zu beobachten? Wie gross ist die Planktonaktivität unter der Eisdecke?

10. Ferner sollte die Strahlung bei den Untersuchungen, besonders im Zusammenhang mit der Planktonaktivität, berücksichtigt werden.

11. Zur weiteren Charakterisierung der Seen wären Untersuchungen des Litorals und Profundals zu empfehlen.

\section{Zusammenfassung}

In den Jahren 1967 bis 1970 wurden in den drei Bergseen Silser See, Silvaplaner See und St.Moritzer See, die rund $1800 \mathrm{~m}$ ü.M. im Oberengadin liegen, je einmal bzw. im Jahre 1968 dreimal Wasserproben erhoben. Der vorliegenden Mitteilung liegen je sechs Probeserien zugrunde. Ziel der Untersuchungen war es, den chemischphysikalischen und den biologischen Charakter der drei Seen zu erfassen.

Untersucht wurden Temperatur, Leitfähigkeit, $\mathrm{pH}$, Transmission, Sauerstoff, Karbonat- und Gesamthärte, Phosphat- und Gesamtphosphor (roh und filtriert), 
Nitrit-, Nitrat-, Ammonium- und Kjeldahl-Stickstoff (roh und filtriert). Ferner wurde das Phytoplankton mit KJK-Lösung fixiert und im Umkehrmikroskop nach Utermöhl ausgezählt. Aus den Individuenzahlen wurde mit Hilfe der berechneten Zellvolumina seine Biomasse bestimmt. Im Silser und St. Moritzer See wurde ausserdem die Primärproduktion mittels der $\mathrm{C}_{14}$-Methode gemessen.

Silser und Silvaplaner See verhielten sich thermisch ähnlich. Beide sind relativ stark durchflutet und stehen unter dem Einfluss von Schmelz- und Gletscherwasser. Der St.Moritzer See zeigte in 20 bis $30 \mathrm{~m}$ Tiefe eine ausgeprägte Sprungschicht. Der Silser See war durchwegs wärmer als die beiden anderen Seen, obgleich er der erste und höchstgelegene der drei aufeinanderfolgenden Seen ist. Möglicherweise hängt dies mit einer stärkeren Windeinwirkung zusammen.

Die Leitfähigkeit war der Gesteinsunterlage (Granite, Gneise, Ophiolithe) entsprechend in allen drei Seen gering (etwa $85-100 \mu \mathrm{s} / \mathrm{cm}$ an der Oberfläche). Der St. Moritzer See unterschied sich von den beiden anderen vor allem durch eine auffallende Zunahme der Leitfähigkeit im Hypolimnion.

Während Silser und Silvaplaner See am Ende der Sommerstagnationsperiode nur einen schwachen Sauerstoffschwund im Hypolimnion aufwiesen, war derselbe im St. Moritzer See stark, so dass der Sauerstoffgehalt im Hypolimnion meist weniger als $1 \mathrm{mg} / \mathrm{l}$ betrug.

Ebenso eindeutig waren die Unterschiede zwischen Silser und Silvaplaner See und St.Moritzer See bezüglich Phosphat- und Gesamtphosphor. Zur Zeit der Zirkulation betrug der Gehalt an Phosphat-Phosphor $5 \mu \mathrm{g} / \mathrm{l}$ in den beiden ersten und $35 \mu \mathrm{g} / \mathrm{l} \mathrm{im}$ St. Moritzer See, der Gesamtphosphor 11-13 bzw. $43 \mu \mathrm{g} / \mathrm{l}$.

Der unterschiedliche Charakter der drei Engadiner Seen kommt, obgleich weniger deutlich, auch in den Stickstoffwerten (Nitrat- und Gesamtstickstoff) zum Ausdruck. Sie waren zur Zeit der Zirkulation etwas höher im St. Moritzer See.

Die Biomasse schwankte in den je sechs Probeserien zwischen 0,06 und 1,85 mg/1 (Silser See), 0,06 und 1,81 mg/l (Silvaplaner See) und 0,04 und 1,97 mg/1 (St. Moritzer See), wobei im Silser See $1 \mathrm{mg} / 1$ nur einmal, im Silvaplaner See zweimal und im St. Moritzer See viermal überschritten wurde.

Die Assimilationsrate ergab von je fünf Probeserien 7-647 $\mathrm{mg} \mathrm{C}_{\text {ass }} / \mathrm{m}^{2} / \mathrm{Tag}$ im Silser See und 439-830 $\mathrm{mg} \mathrm{C}_{\text {ass }} / \mathrm{m}^{2} /$ Tag im St. Moritzer See. Die drei Seen können nach VOLLENWEIDER [17] wie folgt beurteilt werden:

Silser und Silvaplaner See sind bezüglich Sauerstoff, Phosphat- und Gesamtphosphor, Nitrat- und Gesamtstickstoff als oligotroph zu betrachten. Sie gehören wohl zu den saubersten Seen der Schweiz. Der St. Moritzer See, bis zum Jahre 1968 beeinflusst durch die direkte Einleitung der häuslichen Abwässer von St.Moritz, muss bezüglich Sauerstoffgehalt, Phosphat- und Gesamtphosphor, weniger aber aufgrund seines Gehaltes an Nitrat, seiner Biomassewerte und Assimilationsraten, als eutroph bezeichnet werden.

\section{SUMMARY}

Between 1967 and 1970 water samples were taken from the three mountain lakes, the Lakes of Sils, Silvaplana and St. Moritz in the Upper Engadin, which lie at an altitude of approximately $1800 \mathrm{~m}$. Sampling was carried out once a year and three times in 1968. The following study is based on 6 sampling series and is an attempt to evaluate the chemical, physical and biological properties of the three lakes. 
The temperature, conductivity, $\mathrm{pH}$, transmission, oxygen, carbonate content and total hardness, phosphates and total phosphorus (crude and filtered), nitrits, nitrates, ammonium and Kjeldahl nitrogen (crude and filtered) were measured. The phytoplankton was fixed with a JKJ solution and counted under a reversible microscope according to the Utermöhl method. The biomass was determined on the basis of the count and the cell volume calculated. In addition, the primary production of the Lakes of Sils and St. Moritz were also determined by means of the $\mathrm{C}_{\mathbf{1 4}}$ method.

The thermal characteristics of the Lakes of Sils and Silvaplana were very similar. In both the flow is relatively strong and both are influenced by icy glacier water. At a depth of 20 to $30 \mathrm{~m}$ the Lake of St. Moritz has a distinct thermocline. The Lake of Sils was warmer than the others although it is the first and highest of the chain of lakes. This might be due to the effects of the wind which are much stronger here.

As can be expected from the underlying rock (granite, gneiss, ophiolith) the conductivity of all three lakes is insignificant (about 85 to $100 \mu \mathrm{s} / \mathrm{cm}$ on the surface). The Lake of St. Moritz differed from the others in that the conductivity increased significantly in the hypolimnion.

While at the end of the summer stagnation period the Lakes of Sils and Silvaplana showed only a slight decline in the oxygen content in the hypolimnion, it was so great in the Lake of St. Moritz that the oxygen content in the hypolimnion was usually lower than $1 \mathrm{mg} / \mathrm{l}$.

The difference in the phosphate and total phosphorus content of the Lakes of Sils and Silvaplana on the one hand and St. Moritz on the other was just as unequivocal. During the overturn period the phosphate-phosphorus content of the first two lakes was $5 \mu \mathrm{g} / 1$ and $35 \mu \mathrm{g} / \mathrm{l}$ for the Lake of St. Moritz, while total phosphorus content was 11-13 $\mu \mathrm{g} / 1$ and $43 \mu \mathrm{g} / \mathrm{l}$ respectively.

The different characteristics of the three lakes can also be seen from the nitrogen values (nitrates and total nitrogen) although less clearly. During the overturn the figures for the Lake of St. Moritz were somewhat higher.

In the six sampling series carried out for each lakes, the biomass varied between 0.06 and $1.85 \mathrm{mg} / 1$ (Lake of Sils), 0.06 and $1.81 \mathrm{mg} / \mathrm{l}$ (Lake of Silvaplana) and 0.04 and $1.97 \mathrm{mg} / 1$ (Lake of St. Moritz); $1 \mathrm{mg} / 1$ was exceeded only once in the Lake of Sils, twice in the Lake of Silvaplana and four times in the Lake of St. Moritz.

The 5 sampling series per lake regarding the rate of assimilation showed $7-647 \mathrm{mg} \mathrm{C} \mathrm{C}_{\text {ass }} / \mathrm{m}^{2} / \mathrm{day}$ in the Lake of Sils and $439-830 \mathrm{mg} \mathrm{C}$ ass $/ \mathrm{m}^{2} /$ day in the Lake of St. Moritz.

According to VollenweIder [17], the three lakes can be evaluated as follows:

With regard to oxygen, phosphates, total phosphorus, nitrates and total nitrogen the Lakes of Sils and Silvaplana can be considered oligotrophic. They are among the cleanest lakes of Switzerland. The oxygen balance, phosphate and total phosphorus content, but not the nitrate content, standing crop and assimilation rates of the Lake of St.Moritz, into which domestic sewage had been dumped until 1968, show that it is eutrophic.

\section{LITERATURNACHWEIS}

[1] Bachmann, H., Das Phytoplankton der Pioraseen nebst einigen Beitragen zur Kenntnis des Phytoplanktons schweizerischer Alpenseen, Schweiz. Z. Hydrol. 4, 1-103 (1928).

[2] Borner, L., Die Bodenfauna des St.Moritzer Sees, Arch. Hydrobiol. 8 (1917).

[3] FindenegG, I., Bestimmung des Trophiegrades von Seen nach der Radiocarbonmethode, Die Naturwissenschaften 57, 365 (1964).

[4] GächTER, R., Phosphorhaushalt und planktische Primärproduktion im Vievwaldstätter See (Horwer Bucht), Schweiz. Z. Hydrol. 30, 2-66 (1968).

[5] Messikommer, E., Materialien zur Algenkunde des Obevengadins, Schweiz. Z. Hydrol. 27, 1.16-166 (1965).

[6] Pavoni, M., Die Bedeutung des Nannoplanktons im Vergleich zum Netzplankton, Schweiz. $Z$. Hydrol. 25, 219-341 (1963).

[7] Pavoni, M., Beziehung zwischen Biomasse und Stickstoffgehalt des Phytoplanktons und die daraus ableitbare Anwendung der Bestimmungsmethoden für die Praxis, Schweiz. Z. Hydrol. 31, 110-127 (1969). 
[8] Pechlaner, R., Die Finsterthalev Seen (Kiihtai, Österreich). I. Morphometrie, Hydrographie, Limnophysik und Limnochemie, Arch. Hydrobiol. 62, 165-230 (1966).

[9] Pechlaner, R., Die Finsterthaler Seen (Kühtai, Österveich). II. Das Phytoplankton, Arch. Hydrobiol. 63, 145-193 (1967).

[10] Rodne, W., Sulla produzione di fitoplankton in laghi trasparenti di alta montagna, Mem. Ist. Ital. Idrobiol. 15, 21-28 (1962).

[11] Rodнe, W., Standard Correlations Between Pelagic Photosynthesis and Light, Mem. Ist. Ital. Idrobiol. 18, 365-381 (1965).

[12] Rodhe, W., HobBie, J. E., und WRIGHT, R. T., Phototrophy and Heterotrophy in High Mountain Lakes, Verh. int. Verein. theor. angew. Limnol. 16, 302-313 (1966).

[13] Saraceni, C., Il fabbisogno in fosforo e ferro nella coltura di tre specie di Diatomee planctoniche del Lago Maggiore (Tabellavia fenestrata Kïtz., Fragilaria crotonensis Kitton, Asterionella formosa Hassall), Mem. Ist. Ital. Idrobiol. 20, 117-131 (1966).

[14] Schmassmann, H., Die Bodenfauna hochalpiner Seen, Arch. Hydrobiol., Suppl.-Bd. 3 (1920).

[15] Schmassmann, W. und $\mathrm{H}$., Chemische Untersuchungen im St.Moritzer See, Schweiz. Z. Hydrol. 10, 21-35 (1948).

[16] Thомаs, E. A., Empirische und experimentelle Untersuchungen zur Kenntnis der Minimumstoffe in 46 Seen der Schweiz und angrenzender Gebiete, Monatsbulletin des Schweiz. Ver. von Gas- und Wasserfachmännern, Nr. 2 und 3, 1-15 (1953).

[17] VollenweIder, R., Die wissenschaftlichen Grundlagen der Seen- und Fliessgewässeveutrophierung, unter besondever Berücksichtigung des Phosphors und des Stickstoffs als Eutrophierungsfaktoren, Organization for Economic Cooperation and Development (Paris 1968).

Anschrift der Autorin:

Frau Dr. Marianne Bosli-Pavoni, Zielackerstr. 4, CH-8603 Schwerzenbach. 